\title{
Where are Cortical Lesions Responsible for Opercular Syndrome?
}

\author{
Jun-fa Wu, Yi Wu, Feng Zhang, Han-qiu Liu, Yong-shan Hu
}

Can J Neurol Sci. 2013; 40: 97-100

Opercular syndrome (OS), also known as Foix-ChavanyMarie syndrome, is clinically characterized by partial paralysis of facial, lingual, masticatory, and pharyngeal muscles, with preservation of reflexive, automatic, and emotional innervation $^{1,2}$. As a relatively rare cause for dysphagia and dysarthria, OS has been increasingly reported in recent years ${ }^{1,2}$. To the authors' knowledge, only a few studies have explored the pathophysiology of the disease.

Previous studies have suggested that OS is related to dysfunctions of the opercular and perisylvian regions ${ }^{1,2}$, and OS has been classified according to the site of cortical lesions: bilateral anterior opercular, opercular-subopercular, subopercular, unilateral, or posterior ${ }^{1}$. However, this presumption was primarily based on clinical observations and computed tomography (CT) or magnetic resonance imaging (MRI) findings that identified lesions in OS patients and was not confirmed by other methods. The diagnosis of OS is usually based on clinical symptoms and there are no well-established imaging criteria ${ }^{1,2}$.

In the present study, we reported a patient with OS due to traumatic brain injury and used functional MRI (fMRI) to observe eight healthy volunteers' cortical activation patterns while tapping the tooth and moving tongue tip backwards along the palate, which were the two characteristic affected movements in OS. Finally, we sought to determine cortical lesions responsible for OS, and provided some information for establishing the diagnostic criteria of OS.

\section{Materials AND Methods}

\section{Case report}

A 30-year-old man suffered a severe traumatic brain injury in July of 2009. Bilateral removal of an intracranial hemorrhage and decompressive craniectomy were performed immediately after the accident. After surgery the patient gradually recovered, but he continues to have problems swallowing. He could not utter a word, and maintained communication with other people by gestures and eye contact. While eating foods, the patient needed to hyperextend his neck, and the food moved to the oropharynx due to its gravity.

Two years after his initial injury, the patient came to our outpatient clinic for a follow-up examination. He had no auditory comprehension deficit and could obey commands. He could only give forth indistinguishable sounds by gutturophony, and had severe drooling. The patient could not tap his teeth or move his tongue, but involuntary facial movements were preserved. His gag reflex and jaw jerk were present. His limb muscle strength was normal, and his gait was also intact. His activities of daily living score was 100 points. A CT scan of the head showed focal encephalomalacia in bilateral rolandic opercula (ROs) and precentral gyri (PCGs; Figure 1). Based on his clinical presentation and CT scan, the diagnosis of OS was established.

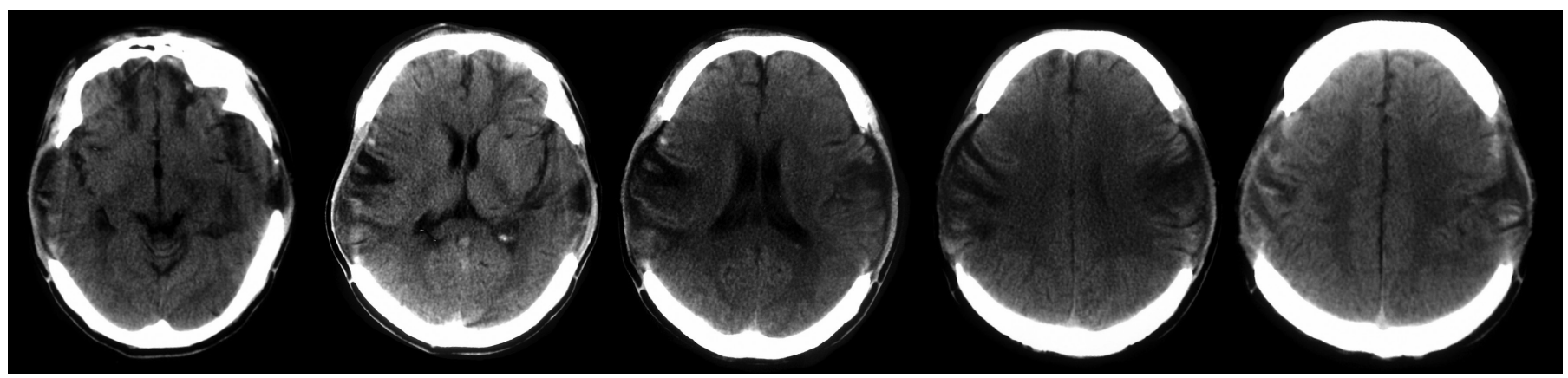

Figure 1: Patient's CT scan two years after traumatic brain injury showing areas of hypodense signals on bilateral rolandic opercula and adjacent precentral gyri.

\footnotetext{
From the Department of Rehabilitation (JFW, YW, FZ, YSH), Department of Radiology (HQL), Huashan Hospital, Fudan University, Shanghai, P. R. China.

Received May 2, 2012. Final ReVisions Submitted July 12/12.

Correspondence to: Yong-Shan Hu, Department of Rehabilitation Medicine, Hua Shan Hospital, Fudan University, Wulumuqi Middle Road 12, Shanghai 200040, China Email: doctorhuyongshan@126.com.
} 

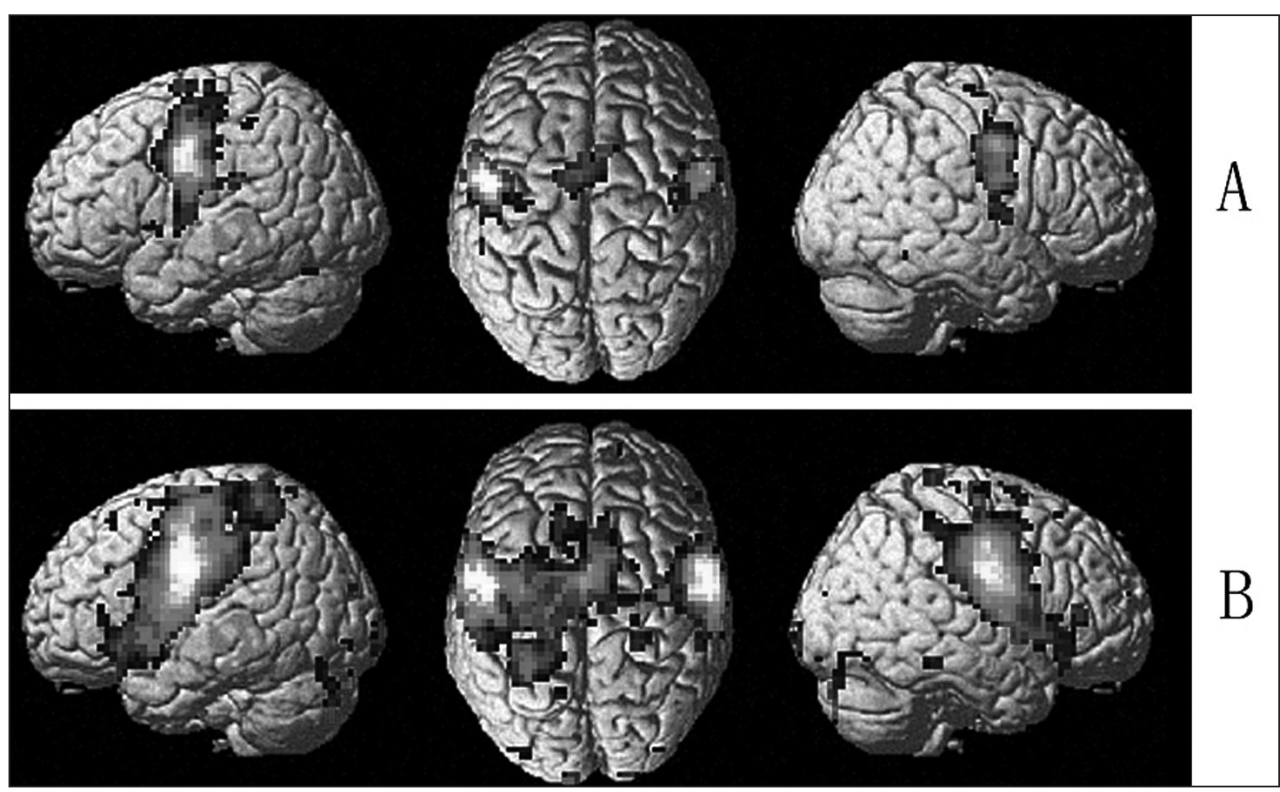

Figure 2: Group maps generated from random effect analysis showing activations during tapping teeth-related activation (A) and moving tongue tip backwards along the palate-related activation (B). In $A$ and $B$, the left and right images represent the left and right hemisphere, respectively. The image in the middle of the figure represents an overhead view.

\section{fMRI study}

\section{Healthy participants}

Eight healthy right-handed volunteers, four men and four women, were included in the present study. Their average age was $31 \pm 8.1$ years (age range: $21-46$ years). The participants were free of neurological or psychiatric disorders. They denied history of motor and sensory disorders. Informed consent was obtained from each subject prior to the experiment.
Prior to the measurement, all subjects were trained to correctly do the two tasks without any other unnecessary motion. During the experiment, all subjects were placed supine and their eyes were closed. 'Start' and 'stop' acoustic signals were given during the acquisition.

\section{fMRI data analysis}

Functional MRI (fMRI) data were analyzed using Statistical Parametric Mapping (SPM)-5 software (www.fil.ion.ucl.ac.uk/

\section{fMRI data acquisition}

The imaging study was performed on a GE 3.0-T MR scanner. The blood oxygen level dependent (BOLD) images were acquired using echo-planar imaging with the following parameters: Field of view $24 \times 24 \mathrm{~cm}$; matrix $64 \times 64$; slice thickness $5 \mathrm{~mm}$; TR $3 \mathrm{~s}$; TE $35 \mathrm{~ms}$. In addition, 25 slices were obtained from a single session.

\section{Task paradigm}

The inability to chew and move the tongue are characteristic symptoms of $\mathrm{OS}^{1,2}$. Chewing mainly includes the two components of tapping the teeth and moving the tongue. The former is that the upper and lower teeth repetitively tap against each other when closing and opening the jaw in chewing. We employed two tasks, namely self-paced tapping the teeth or self-paced moving the tongue tip backwards along the palate. Both task sessions were designed in a block manner (three rest and three task blocks, every task block for 30s).
Table: Location of significant activations

\begin{tabular}{|c|c|c|c|c|c|c|c|c|}
\hline \multirow{3}{*}{ Brain area } & \multicolumn{4}{|c|}{ Tapping the teeth } & \multicolumn{4}{|c|}{ Moving the tongue tip backwards along the palate } \\
\hline & \multirow[t]{2}{*}{ Talairach $(\mathrm{x}, \mathrm{y}, \mathrm{z})$} & \multicolumn{2}{|c|}{ Voxel- level } & \multirow[t]{2}{*}{$\mathrm{K}_{\mathrm{E}}$} & \multirow[t]{2}{*}{ Talairach $(\mathrm{x}, \mathrm{y}, \mathrm{z})$} & \multicolumn{2}{|c|}{ Voxel- level } & \multirow[t]{2}{*}{$\mathrm{K}_{\mathrm{E}}$} \\
\hline & & $\mathrm{T}$ & $\mathrm{ZE}$ & & & $\mathrm{T}$ & ZE & \\
\hline $\mathrm{L}-\mathrm{PCG}$ & $-54,-6,39$ & 14.38 & $>8$ & 790 & $-51,-9,30$ & 20.46 & $>8$ & 5445 \\
\hline \multirow[t]{2}{*}{ L - PCG (BA4) } & $-54,-6,51$ & 13.14 & $>8$ & & $-54,-6,45$ & 18.42 & $>8$ & \\
\hline & & & & & $-51,-6,52$ & 16.08 & $>8$ & \\
\hline \multirow[t]{2}{*}{ R - PCG (BA6) } & $45,-6,33$ & 10.24 & $>8$ & 409 & $57,-9,27$ & 17.26 & $>8$ & 1884 \\
\hline & $60,-6,45$ & 10.05 & $>8$ & & $57,-9,42$ & 15.73 & $>8$ & \\
\hline R - PCG & $57,-3,24$ & 8.14 & 7.61 & & $45,-12,30$ & 14.53 & $>8$ & \\
\hline L - MFG (BA6) & $-12,-9,69$ & 7.74 & 7.28 & 170 & & & & \\
\hline R - SFG (BA6) & $9,9,54$ & 5.89 & 5.67 & & & & & \\
\hline $\mathrm{L}-\mathrm{CTU}$ & & & & & $-21,-87,-30$ & 10.14 & $>8$ & 96 \\
\hline L - CISLL & & & & & $-27,-81,-39$ & 8.44 & 7.83 & \\
\hline $\mathrm{R}-\mathrm{CTU}$ & & & & & $36,-84,-24$ & 7.78 & 7.31 & 73 \\
\hline R - CISLL & & & & & $30,-84,-36$ & 7.63 & 7.19 & \\
\hline $\mathrm{L}-\mathrm{RO}$ & $-56,0,9$ & 5.84 & 5.37 & 70 & $-57,0,7$ & 9.24 & $>8$ & 88 \\
\hline $\mathrm{R}-\mathrm{RO}$ & $60,0,6$ & 5.79 & 5.16 & 50 & $59,3,6$ & 8.8 & $>8$ & 80 \\
\hline
\end{tabular}

$(\mathrm{P}<0.05$ corrected at the cluster level) during tapping the teeth and moving the tongue tip backwards along the palate in eight healthy subjects. L, left; R, right; PCG, precentral gyrus; MFG, medial frontal gyrus; SFG, superior frontal gyrus; CTU, cerebellum tubers; CISLL, cerebellum inferior semilunar lobules; RO, rolandic operculum; KE, cluster level. 


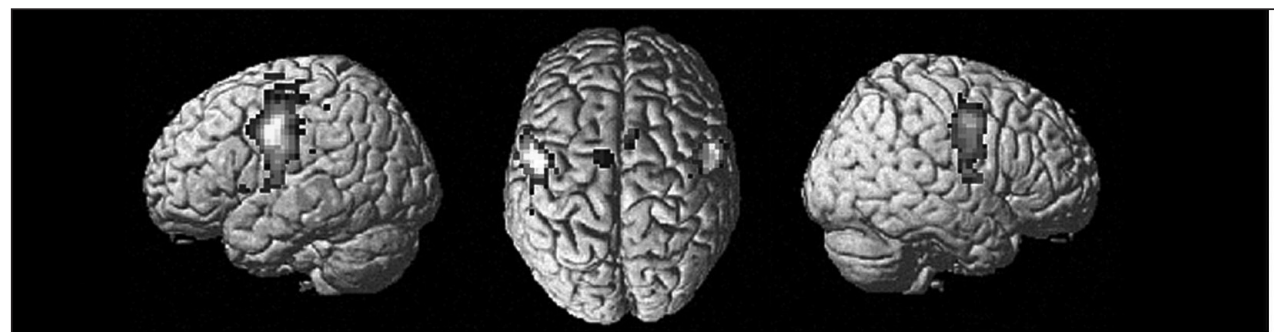

A

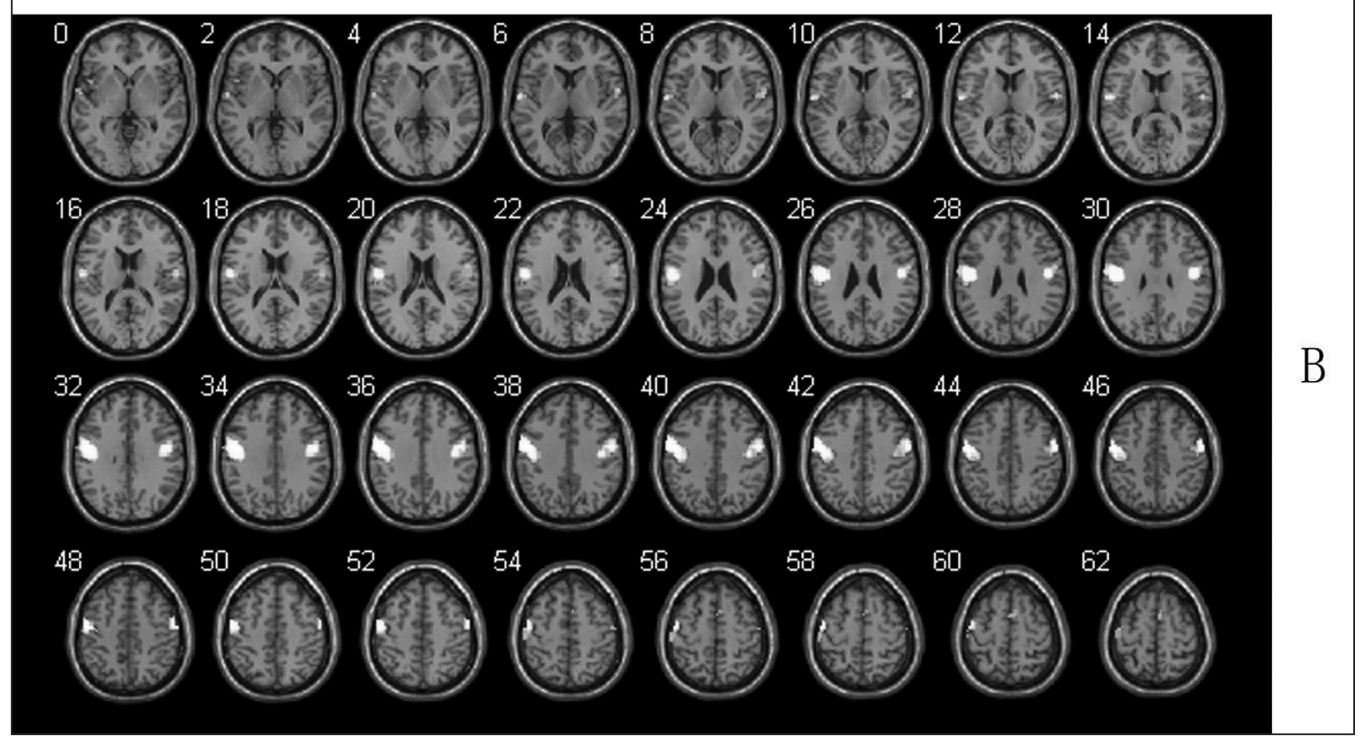

Figure 3: The activated areas common to the two tasks from conjunction analysis. A: Rendered on a 3D brain template. B: The functional images viewed by xjView Microsoft.

spm). All images were realigned and then normalized to the echo-planar imaging template, and functional data were smoothed with a Gaussian kernel (full width at half maximum $=$ $6 \times 6 \times 6 \mathrm{~mm})$. Statistical parametric maps were obtained and voxels were considered significant at a threshold of $\mathrm{P}<0.05$ FWE- corrected. The commonly activated areas for the two event-related tasks were calculated by conjunction analysis.

\section{RESUlts}

The task of tapping the teeth was related to significant increases in the BOLD signal in the bilateral PCGs, extending down to the upper part of bilateral ROs (Figure 2A). In addition, the BOLD signal increases were observed in left medial frontal gyri and right superior frontal gyri. During the tongue-tip movement task, significant activations were observed in bilateral PCGs and adjacent ROs. In addition, bilateral cerebellar tubers and inferior semilunar lobules of the cerebellum were also activated (Figure 2B). The locations of the most significant activation are summarized in the Table, which lists the anatomical regions with maximal $\mathrm{t}$ values and $\mathrm{Z}$ scores in clusters and the coordinates as given in the Talairach and Tournoux atlas.

The conjunction analysis identified two regions common to the two tasks. The two regions were mainly located at bilateral PCGs $(\mathrm{x}=-54, \mathrm{y}=-3, \mathrm{z}=36$, with $\mathrm{T}=10.21$; and $\mathrm{x}=57, \mathrm{y}=$
$-6, \mathrm{z}=45$, with $\mathrm{T}=8.80)$ and the adjacent ROs $(\mathrm{x}=-57, \mathrm{y}=-8$, $\mathrm{z}=9$, with $\mathrm{T}=6.20$; and $\mathrm{x}=59, \mathrm{y}=-6, \mathrm{z}=9$, with $\mathrm{T}=5.44$; Figure 3$)$. The common activated areas were nearly the same as all visible lesions of the patient on CT, which were also located at bilateral ROs and the adjacent PCGs.

\section{DISCUSSION}

In our study, the patient presented with bilateral central faciolinguo-velo-pharyngeo- masticatory paralysis with automaticvoluntary dissociation. The clinical findings are consistent with the core features of $\mathrm{OS}^{1,2}$. The cause of OS in the patient is most likely traumatic brain injury.

In the present fMRI study, we found that the activated areas of two tasks in healthy volunteers were mainly located at bilateral ROs and adjacent PCGs. These areas were consistent with the brain lesions of the OS patient on CT. In previous studies, the cortical lesions were also within the common activated areas ${ }^{1,2}$. Based on previous reports and our findings, we propose that bilateral partial damage to the common activated areas were probably responsible for the development of OS. For some patients, OS might be attributed to subcortical lesions (i.e., the opercular-subopercular and subopercular types). One plausible explanation might be that efferent impulses to peripheral muscles from the cortical activated areas were interrupted by these lesions. Additionally, the ROs and PCGs 
activations during tapping the teeth or moving the tongue are also consistent with the results reported by Martin ${ }^{3}$ and Sessle ${ }^{4}$. Based on these observations, damage to bilateral ROs or PCGs might affect the planning and execution of facio-glossomasticatory movement, leading to OS.

To some extent, our findings also support the previously established neural control mechanism of chewing and tongue movement ${ }^{2}$. These are the most common motor function impairments in OS patients ${ }^{1}$. Chewing is mainly performed by masticatory, buccinator, and glossal muscles. Tapping the teeth, as a primary component of chewing, requires contraction of the masticatory muscles. In contrast, the movement of the tongue is produced by the glossal muscles. According to classic neuroanatomy, unilateral masticatory muscles are innervated by ipsilateral masticator nuclei, which receive motor input from the bilateral cerebral cortex. The unilateral glossal muscle only receives innervation from the ipsilateral hypoglossal nucleus. The ipsilateral hypoglossal nucleus only receives motor input from the contralateral cerebral cortex ${ }^{2}$. Based on these neural projections, the inability to chew or move the tongue should be caused by damage to the bilateral motor cortex or efferent fibers from the bilateral motor cortex, controlling the masticatory and glossal muscles. If the unilateral motor cortex is damaged, the contralateral motor cortex is capable of compensating the function of the affected motor cortex.

Previous studies showed that some patients presented with OS after epileptic attacks ${ }^{5}$. Interestingly, their brain CT or MRI did not show any focal lesion. Some of these patients may recover spontaneously after epileptic attack. Based on these facts and our findings, we propose that epileptic seizure might lead to temporary functional inhibition of bilateral ROs or adjacent PCGs. Both organic injury and functional inhibition may lead to the development of OS. Therefore, the diagnosis of OS should not only be based on clinical symptoms, but also require radiologic evidence of damage to the common activated areas or fiber pathways of the two tasks. If imaging evidence is not available, clinical symptoms and EEG may suggest that patients have epilepsy, which might also suppress the function of bilateral ROs and adjacent PCGs.

\section{Conclusion}

In summary, our results suggest that bilateral ROs and adjacent PCGs may be the cortical center of the neural network that controls the motor function of facial, lingual, masticatory, and pharyngeal muscles. Bilateral damages to these regions might be an important underlying cause of OS in most patients. The association between these regions and OS merits further investigation.

\section{REFERENCES}

1. Thapa L, Paudel R, Rana PVS. Opercular syndrome: Case reports and review of literature. Neurol Asia. 2010;15(2):145-52.

2. Nowak DA., Griebl G, Dabitz R, et al. Bilateral anterior opercular (Foix-Chavany-Marie) syndrome. J Clin Neurosci. 2010;17 (11):1441-2.

3. Martin RE, MacIntosh BJ, Smith RC, et al.Cerebral areas processing swallowing and tongue movement are overlapping but distinct:a functional magnetic resonance imaging study. J Neurophysiol. 2004;92:2428-43.

4. Sessle BJ. Face sensorimotor cortex. Its role and neuroplasticity in the control of orofacial movements. Prog Brain Res. 2011;188: $71-82$.

5. Benninger DH, Mueller SG, Treyer V, et al. Transient epileptic opercular syndrome. Seizure. 2007;16:276-82. 